\title{
SIMPLE TROPHIC MODULES FOR COMPLEX FOOD WEBS
}

\author{
Jordi BASCOMPTE ${ }^{1}$ And CARlos J. Melián ${ }^{2}$ \\ Integrative Ecology Group, Estación Biológica de Doñana, CSIC, Apartado 1056, E-41080 Sevilla, Spain
}

\begin{abstract}
There are two common approaches to food webs. On the one hand, empirical studies have described aggregate statistical measures of many-species food webs. On the other hand, theoretical studies have explored the dynamic properties of simple tri-trophic food chains (i.e., trophic modules). The question remains to what extent results based on simple modules are relevant for whole food webs. Here we bridge between these two independent research agendas by exploring the relative frequency of different trophic modules in the five most resolved food webs. While apparent competition and intraguild predation are overrepresented when compared to a suite of null models, the frequency of omnivory highly varies across communities. Inferences about the representation of modules may also depend on the null model used for statistical significance.
\end{abstract}

Key words: apparent competition; complex networks; food-web models; food webs; intraguild predation; network motifs; omnivory.

\section{INTRODUCTION}

Food webs depict the feeding relationships (who eats whom) in communities. Because of the large number of species and interactions, our knowledge of real food webs is limited. Empirical and theoretical studies have developed in different directions. The bulk of empirical studies embrace whole food webs and describe their organization mainly through aggregate measures such as connectance or compartmentalization (Cohen 1977, Pimm and Lawton 1980, Sugihara et al. 1989, Martinez 1991, Bersier et al. 1999, Pimm 2002). The advantage of this approach is to deal with whole, real food webs. The drawback is that it represents merely a static description.

To explore the dynamic consequences of food-web structure one has to rely on theoretical models, for example on the dynamic study of very simple tri-trophic food chains or trophic modules (Fig. 1a-d). Despite their simplicity, these modules contain the basic distinct types of trophic interrelationships such as omnivory (Fig. 1b), apparent competition (Fig. 1c), and intraguild predation (i.e., killing and eating among potential competitors, Polis and Holt 1992, Arim and Marquet 2004; Fig. 1d). They can be seen as the simplest representations of multitrophic interactions

Manuscript received 24 January 2005; revised 31 May 2005; accepted 3 June 2005. Corresponding Editor: W. S. C. Gurney.

${ }^{1}$ E-mail: bascompte@ebd.csic.es

${ }^{2}$ Present address: National Center for Ecological Analysis and Synthesis, University of California, 735 State Street, Suite 300, Santa Barbara, California 93101 USA.
(Pimm and Lawton 1977, Holt 1996, McCann et al. 1998 ) or the building units of complex food webs (Milo et al. 2002).

Several dynamic results have been obtained with simple modules. For example, theory has emphasized the stabilizing role of omnivory (McCann et al. 1998), although, as pointed out by Pimm (2002:xxvii) "no one has yet applied a food-web null hypothesis and shown that omnivores are as common than [sic] one would expect." To assess the importance of these dynamic results for real food webs, one has to determine how well represented these simple modules are in real food webs (Arim and Marquet 2004). If, for example, omnivory is underrepresented, its potential stabilizing mechanism will hardly be of importance in real communities. In this paper we extend recent research to detect simple motifs in complex networks (Milo et al. $2002,2004)$ to explore the relative frequency of several trophic modules in the five food webs containing the largest number of trophic species and interactions. Our choice of the studied motifs is not dictated by arbitrary reasons but reflects the theoretical research agenda on food webs. Because theoretical ecologists study the dynamics of these modules without knowing their relative importance in real ecosystems, our paper aims at linking two different approaches to food webs. Previous studies in ecology have used a similar approach of comparing a specific pattern in both real food webs and ecological models (some of which are used in here). However, this previous work was based on aggregate measures such as connectance, fraction of species at top, intermediate, and basal trophic levels, and food- 

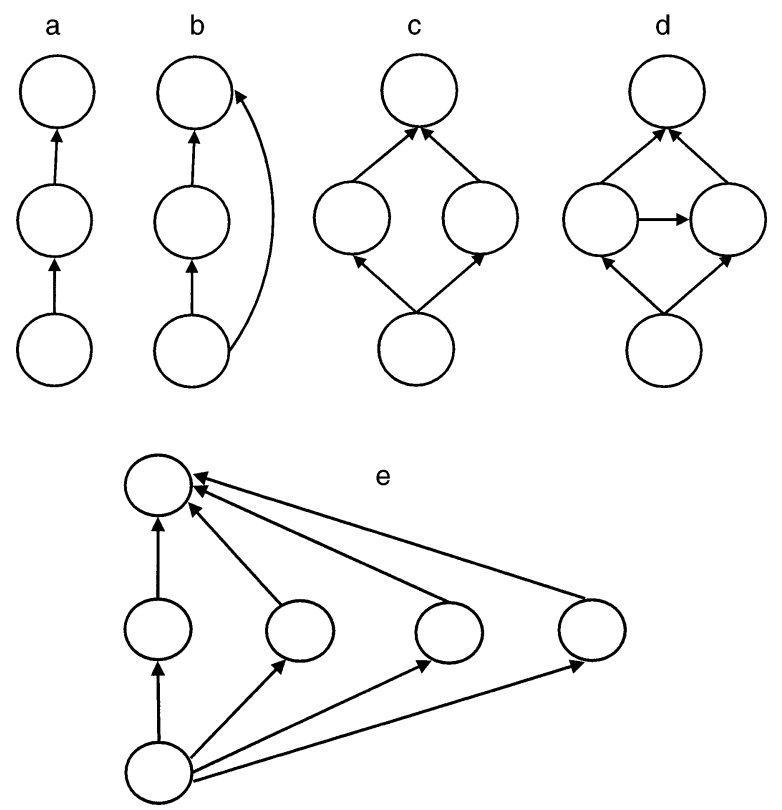

FIG. 1. Trophic modules studied in this paper: (a) simple food chain, (b) omnivory, (c) apparent competition, and (d) intraguild predation. (e) A fraction of the food web containing several interrelated modules. In this example, one can count four simple food chains and six apparent competition modules.

chain length (Cohen et al. 1990, Williams and Martinez 2000, 2004, Cattin et al. 2004). In our present study we move beyond this description to focus on trophic modules that define a higher level of network structure.

\section{Methods}

The five food webs studied are Ythan Estuary (Huxam et al. 1996), Silwood Park (Memmott et al. 2000), El Verde (Reagan and Waide 1996), Little Rock Lake (Martinez 1991), and Caribbean food web (Bascompte et al. 2005). These are the largest food webs compiled so far, with $134,154,156,182$, and 249 trophic species, respectively.

In each food web we count the number of four different trophic modules: simple food chains (FC), omnivory (OMN), apparent competition (APC), and intraguild predation (IGP) (Fig. 1). These are qualitative motifs, that is, there is no information on the strength of each interaction. For a similar study on quantitative modules see the recent study by Bascompte et al. (2005) using a single highly resolved food web. Loops (e.g., A eats B, which in turn eats A) are not considered. Note that in our definition of omnivory the basal species is not necessarily a plant (e.g., it can be an herbivore eaten by both a consumer and a top predator). Also, a note should be added on how the different modules are scored. Our algorithm counts independently each module, and as noted in Fig. 1, some are contained in others. For example, omnivory contains a simple food chain, apparent competition contains two such modules, and intraguild predation contains three FCs and two OMN. This procedure allows us to explore how different modules are interrelated. This way of scoring modules is slightly different than the one used by Milo et al. (2002), who do not consider modules contained in larger modules as independent modules.

Following previous studies (e.g., Milo et al. 2002, Melián and Bascompte 2004), statistical significance is estimated by comparing the observed number of modules to a population of randomizations of the food web. These randomizations are obtained by using a null model, a pattern-generating model that brings flexibility in data analysis that is difficult to attain with conventional statistical tests (Gotelli 2001). Thus, $P$ is the probability of a random replicate having a larger or equal number of modules than the real food web. If $P$ $<0.05$, the number of modules in the food web is significantly higher than expected by chance. On the other hand, if $P>0.95$ the observed number of modules is significantly lower than expected by chance. This is the $P$ value shown in Table 1 . Because results may strongly depend on the null model used as a benchmark, we make a comparative analysis across four different null models:

1) The local rewiring algorithm (LRA; Gale 1957, Connor and Simberloff 1979, Roberts and Stone 1990, Milo et al. 2002). In this algorithm, a pair of directed links, A-B and C-D are randomly selected and rewired in such a way that $\mathrm{A}$ becomes connected to $\mathrm{D}$ and $\mathrm{C}$ to $\mathrm{B}$, provided that none of these links already existed. By performing a large enough number of such changes we have a randomized network in which each species has the same number of ingoing and outgoing edges as the corresponding species in the real food web. We thus account for patterns that could arise just because of a differential number of edges per species. This model has been intensively used in island biogeography and complex networks, and has no biological assumptions. The following three models, on the contrary, make such biological assumptions.

2) The niche model of Williams and Martinez (2000). Each species is now assigned a "niche-value" parameter $\left(n_{i}\right)$ drawn uniformly from the interval $[0,1]$. One may think of this value as a surrogate for body mass. As explicitly defined by Williams and Martinez (2000), species $i$ consumes all species falling in a range $r_{i}$ that is placed by uniformly drawing the center of the range $c_{i}$ from $\left[r_{i} / 2, n_{i}\right]$. The size of $r_{i}$ is assigned by using a beta function to randomly draw values from $[0,1]$ that are corrected so one gets the observed global connectance (see Williams and Martinez [2000] for further details).

3) Our third null model is a sightly different version of the nested-hierarchy model of Cattin et al. (2004) (nested-hierarchy 1 hereafter). While the original model determines the number of prey for each consumer following the niche model, we preserve the observed number of prey for each consumer. The rest of the 
TABLE 1. Number of trophic modules in five large food webs.

\begin{tabular}{|c|c|c|c|c|c|}
\hline \multirow[b]{2}{*}{ Food web } & \multirow{2}{*}{$\begin{array}{l}\text { Observed no. } \\
\text { of modules }\end{array}$} & \multicolumn{2}{|c|}{ Local rewiring algorithm, LRA } & \multicolumn{2}{|c|}{ Niche model } \\
\hline & & Mean (SD) & $P$ & Mean (SD) & $P$ \\
\hline \multicolumn{6}{|c|}{ FC, simple food chain } \\
\hline Ythan & 3284 & 2929 (103) & $<0.0001$ & $1776(170)$ & $<0.0001$ \\
\hline Silwood & 885 & $818(52)$ & NS & $591(70)$ & $<0.0001$ \\
\hline El Verde & 11655 & $13576(359)$ & $>0.9999$ & $10188(695)$ & $<0.01$ \\
\hline LR Lake & 22256 & $22560(478)$ & NS & $22493(1358)$ & NS \\
\hline Caribbean & 28181 & $25739(598)$ & $<0.0001$ & $30738(1841)$ & NS \\
\hline \multicolumn{6}{|l|}{ OMN, omnivory } \\
\hline Ythan & 487 & $470(39)$ & NS & $215(59)$ & $<0.0001$ \\
\hline Silwood & 358 & $238(28)$ & $<0.0001$ & $51(26)$ & $<0.0001$ \\
\hline El Verde & 2817 & 3077 (154) & 0.95 & $2101(306)$ & $<0.0001$ \\
\hline LR Lake & 9654 & $8082(240)$ & $<0.0001$ & $5344(618)$ & $<0.0001$ \\
\hline Caribbean & 9063 & $11512(274)$ & $>0.9999$ & $5637(715)$ & $<0.0001$ \\
\hline \multicolumn{6}{|c|}{ APC, apparent competition } \\
\hline Ythan & 2691 & $677(90)$ & $<0.0001$ & $238(26)$ & $<0.0001$ \\
\hline Silwood & 266 & $190(67)$ & NS & $20(6)$ & $<0.0001$ \\
\hline El Verde & 21431 & $6043(485)$ & $<0.0001$ & $5099(225)$ & $<0.0001$ \\
\hline LR Lake & 77538 & $30025(1462)$ & $<0.0001$ & $17497(493)$ & $<0.0001$ \\
\hline Caribbean & 168095 & $97861(2739)$ & $<0.0001$ & $18545(555)$ & $<0.0001$ \\
\hline \multicolumn{6}{|c|}{ IGP, intraguild predation } \\
\hline Ythan & 603 & $330(81)$ & $<0.01$ & $45(43)$ & $<0.0001$ \\
\hline Silwood & 976 & $207(68)$ & $<0.0001$ & $12(16)$ & $<0.0001$ \\
\hline El Verde & 6594 & $5180(692)$ & $<0.05$ & $2014(825)$ & $<0.0001$ \\
\hline LR Lake & 40554 & $16375(1486)$ & $<0.0001$ & 8537 (2055) & $<0.0001$ \\
\hline Caribbean & 25269 & $22857(1854)$ & NS & $6446(2143)$ & $<0.0001$ \\
\hline
\end{tabular}

Note: For each food web the table reports the observed number of modules, and the estimated number of modules based on 1000 replicates of the models.

model is exactly similar to the nested-hierarchy model (details can be found in Cattin et al. [2004]). Starting with the smallest consumer's niche value, the trophic links are attributed to consumers in a two-stage process. In stage one, prey species of consumer $j$ are randomly chosen among species with rank $<j$. Depending on this randomly chosen prey $i$, two cases are possible: (1) prey $i$ has no consumer and therefore the next prey of consumer $j$ will again be randomly attributed (with rank of prey $<j$ ); (2) prey $i$ already has one or more consumers and therefore consumer $j$ joins the group of species $i$ 's consumers, and the next prey of consumer $j$ is then randomly chosen among the set of prey of this group. However, if the number of prey in the group is too small for choosing all remaining prey of consumer $j$, the remaining prey are again randomly chosen among prey without consumers (with rank $<j$ ). By creating groups of consumers, stage one accounts for phylogenetic constraints. Links attributed to species free of consumers, and links distributed randomly in the second stage, reflect the adaptation of consumers to new prey (Cattin et al. 2004).

4) Nested-hierarchy 2. The previous models assume a homogeneous distribution of consumers and prey in space. As correctly pointed out by Artzy-Randrup et al. (2004), spatial structure imposing a constraint not included in a nonspatial randomization could explain the finding of a significant pattern. To explore this we introduce a modification of the previous null model. All the rules are the same with the following exception in point (2) of the first stage, when assigning additional prey of consumer $j$ once this has joined the group of species $i$ 's consumers. As before, the next prey of consumer $j$ is then randomly chosen among the set of prey of this group. However, if the number of prey in the group is lower than the total number of prey that the predator has, the remaining prey are randomly chosen among the set of consumers that share at least one prey with consumer $j$ (instead of randomly chosen among all prey without consumers with rank $<j$ ). This rule could be relaxed, but in our present study we set the probability of prey assignment among the set of consumers that share at least one prey to 1 . If the group of consumers that share at least one prey with consumer $j$ is too small for choosing all remaining prey of consumer $j$, the remaining prey that could not be attributed are randomly chosen (prey species can have rank $\geq i$ ). Note that this adaptation assumes that consumer $j$ is forced to eat other consumer species with which it shares a prey. This difference accounts for the fact that individuals sharing a common prey are in the same habitats and so have an increased probability to interact among themselves. Thus, the model attempts to capture spatial aggregation of consumers that share prey. As a consequence, it necessarily increases the frequency of IGP.

We generated a population of 1000 replicates for each null model and each food web (we used MATLAB [Mathworks, Natick, Massachusetts, USA]). 
TABLE 1. Extended.

\begin{tabular}{|c|c|c|c|}
\hline \multicolumn{2}{|c|}{ Nested-hierarchy 1 model } & \multicolumn{2}{|c|}{ Nested-hierarchy 2 model } \\
\hline Mean (SD) & $P$ & Mean (SD) & $P$ \\
\hline $\begin{array}{r}1653(87) \\
651(41) \\
9388(514) \\
18638(1404) \\
25189(841)\end{array}$ & $\begin{array}{l}<0.0001 \\
<0.0001 \\
<0.0001 \\
<0.05 \\
<0.0001\end{array}$ & $\begin{array}{r}1558(118) \\
515(33) \\
9567(1069) \\
23098(1738) \\
20620(1766)\end{array}$ & $\begin{array}{c}<0.0001 \\
<0.0001 \\
<0.05 \\
\quad \text { NS } \\
<0.0001\end{array}$ \\
\hline $\begin{array}{c}144(31) \\
14(6) \\
1757(209) \\
5818(659) \\
8624(719)\end{array}$ & $\begin{array}{l}<0.0001 \\
<0.0001 \\
<0.001 \\
<0.001 \\
\quad \text { NS }\end{array}$ & $\begin{aligned} 588(72) \\
164(31) \\
3726(393) \\
10763(1348) \\
10487(690)\end{aligned}$ & $\begin{array}{c}0.95 \\
<0.0001 \\
>0.9999 \\
\text { NS } \\
>0.9999\end{array}$ \\
\hline $\begin{aligned} 540(71) \\
240(28) \\
8133(1452) \\
23314(2823) \\
45505(4535)\end{aligned}$ & $\begin{array}{l}<0.0001 \\
\text { NS } \\
<0.0001 \\
<0.0001 \\
<0.0001\end{array}$ & $\begin{array}{c}854(158) \\
234(29) \\
10417(2204) \\
24739(9912) \\
58808(6489)\end{array}$ & $\begin{array}{l}<0.0001 \\
\quad \mathrm{NS} \\
<0.0001 \\
<0.0001 \\
<0.0001\end{array}$ \\
\hline $\begin{aligned} 22 & (15) \\
1 & (1) \\
1420 & (423) \\
8289 & (2707) \\
14890 & (2971)\end{aligned}$ & $\begin{array}{l}<0.0001 \\
<0.0001 \\
<0.0001 \\
<0.0001 \\
<0.0001\end{array}$ & $\begin{aligned} 381 & (147) \\
50 & (25) \\
6905 & (2119) \\
39967 & (12617) \\
16087 & (4382)\end{aligned}$ & $\begin{array}{c}\mathrm{NS} \\
<0.0001 \\
\mathrm{NS} \\
\mathrm{NS} \\
<0.05\end{array}$ \\
\hline
\end{tabular}

\section{RESULTS}

The frequency of each of four modules in each of five food webs is summarized in Table 1. The bulk of cases are significant. In apparent competition (APC) and intraguild predation (IGP) these differences are always in the direction of having a larger number of modules than expected by chance (see also Arim and Marquet [2004] for IGP). These two modules may thus be very relevant for complex food webs. However, omnivory (OMN) shows a higher variability of cases. While it is found less often than expected in El Verde and Caribbean (using both the local rewiring algorithm [LRA] and the nested hierarchy 2), and in Ythan (using the nested hierarchy 2), omnivory is overrepresented in Silwood Park (using both LRA and nested hierarchy 2) and Little Rock Lake (using LRA). Thus, the frequency of omnivory seems to be highly dependent on the specific food web.

Because simple food chains (FCs) are contained in several modules (see Fig. 1a-d) one would conclude that the number of FCs is always higher than the number of other modules. However, Table 1 shows that this is not necessarily the case. For example, in Silwood Park the number of IGP (976) is higher than the number of FCs (885). This is hard to understand if we look only at the scale of the isolated modules. To obtain an answer we need to bridge between such modules and the full food web by looking at how they are interrelated. Fig. 1e shows an example of how the number of combinations of FCs giving place to APC exceeds the number of FCs. This modular redundancy has never been explored, and may have implications for foodweb structure and dynamics.

By comparing across null models one can assess to what extent the previous results depend on the specific null model used. Some results are robust across null models. For example, all four null models reach the same conclusion about the relative frequency of apparent competition in all food webs but in Silwood Park for the niche model. Although to a lesser extent, there is also a high concordance in conclusions about intraguild predation among the four null models. Other results, however, strongly depend on the null model used. For example, LRA and nested-hierarchy 2 both show overrepresentation of omnivory in some food webs and underrepresentation in others, while using the niche and the nested-hierarchy 1 models, one would conclude that omnivory is always overrepresented except in Caribbean for the nested-hierarchy 1 . When looking specifically at which food web has more or less omnivory than expected by chance, and comparing the LRA and nested-hierarchy 2 , in three cases out of five both models predict the same result (always more omnivory or always less omnivory). Similarly, in three out of four null models, one concludes that Silwood Park is the only food web in which the frequency of apparent competition is what we would expect by chance.

\section{DISCUSSION}

Omnivory has been proved to be a stabilizing factor in trophic interactions (McCann and Hastings 1997, Fagan 1997). Interestingly enough, our results suggest 
that the relative importance of this stabilizing factor greatly varies across food webs (using the local rewiring algorithm [LRA] and the nested-hierarchy 2). It is not easy to explain this differential frequency of omnivory on the basis of any qualitative property of the whole food web. For example, both aquatic and terrestrial food webs can have either a larger or lower number of omnivory modules (see Table 1). A close inspection of species composition may be necessary to explain this variability in the frequency of omnivory.

Theoretical work has found that intraguild predation (IGP) can lead to a variety of alternative steady states and to complex dynamics (limit cycles and chaos) associated with the time lags and triangular loops (Polis and Holt 1992). For example, the effects of the elimination of a species can produce counterintuitive results depending on the relative strengths of direct and indirect effects of IGP (Polis and Holt 1992). This dynamic complexity has been found to be responsible for inappropriate fishery management (e.g., in the addition of exotics as food for existing species, Polis and Holt 1992). The overrepresentation of intraguild predation in real food webs found here (except when using the nested-hierarchy 2)(see also Arim and Marquet 2004) suggests that this dynamic complexity may be common in real communities.

Previous work has partially analyzed the frequency of a specific type of module in poorly resolved food webs, but no global study such as the present one has been done so far. For example, Arim and Marquet (2004) analyzed the relative occurrence of intraguild predation, and Milo et al. (2002) examined several network motifs in a data set of biological and electronic networks. Three of these motifs are equivalent to our simple food chain, apparent competition, and omnivory. However, only two of the networks here studied were analyzed by Milo et al. (2002) (together with a set of smaller food webs). The results of Milo et al. regarding food chains (FCs) and apparent competition (APC) are difficult to compare because of the different scoring methods. However, results for omnivory are directly comparable. Milo et al. found omnivory underrepresented, which is what we found in only half the cases (when using the LRA, the same null model they used). This difference may be due to the fact that we are studying larger food webs (even in the two food webs overlapped in both studies, i.e., Ythan and Little Rock Lake, we are analyzing more resolved versions with as many as twice the number of nodes). Our results for these two common food webs contrast with the findings of Milo et al.: in one case omnivory is overrepresented and in the other case there are no significant differences (again, using the LRA). This proves that the level of aggregation of food webs may change results on structure, at least for some modules such as omnivory (although this is not found for the other modules).
Polis and colleagues emphasized the very complex nature of real food webs, with a high fraction of omnivory and other complex relationships (Polis 1991, Polis and Strong 1996). In contrast, Williams and Martinez (2004) questioned this claim by assigning species to an unambiguous trophic level, and finding a relatively low degree of omnivory. However, these previous papers looked at the number of omnivores, while here we look at the frequency of omnivory modules. For example, there could be a single omnivorous species but that species could be involved in a large number of modules. From the point of view of the frequency of omnivorous modules, our results confirm both opposing perspectives, since the role of omnivory strongly depends on the particular food web (when assessed by means of the LRA and nested-hierarchy 2), and level of aggregation (compared with previous studies). When looking at intraguild predation and apparent competition, our results confirm Polis' (1991) view of food webs as complex entities.

To sum up, one can conclude that the bulk of modules are overrepresented in real food webs, that omnivory shows a high degree of variability across food webs, and that inferences depend on the level of aggregation and the null model used. At the end, one has to conclude that null models are only a general reference, and that a suite of them should be used to be able to infer robust conclusions.

Our exploration on the frequency of simple modules is only a first step to address how complex food webs are built up. An interesting follow-up to the present paper would be to explore the various ways these simple modules are interrelated among themselves to form real food webs, and, specifically, the observed modular redundancy.

\section{ACKNOWLEDGMENTS}

We thank P. Jordano, M. A. Fortuna, and the rest of the Integrative Ecology Group for interesting comments, and P. Sosa for computing facilities. Funding was provided by the Spanish Ministry of Science and Technology (Grant REN2003-04774 to J. Bascompte and Ph.D. Fellowship FP2000-6137 to C. J. Melián), and the European Heads of Research Councils and the European Science Foundation through an EURYI award (to J. Bascompte).

\section{Literature Cited}

Arim, M., and P. A. Marquet. 2004. Intraguild predation: a widespread interaction related to species biology. Ecology Letters 7:557-564.

Artzy-Randrup, Y., S. J. Fleisman, N. Ben-Tal, and L. Stone. 2004. Comment on "Network motifs: simple building blocks of complex networks" and "Superfamilies of evolved and designed networks." Science 305:1107.

Bascompte, J., C. J. Melián, and E. Sala. 2005. Interaction strength combinations and the overfishing of a marine food web. Proceedings of the National Academy of Sciences (USA) 102:5443-5447.

Bersier, L. F., P. Dixon, and G. Sugihara. 1999. Scale-invariant or scale-dependent behavior of the link density property in food webs: A matter of sampling effort? American Naturalist 153:676-682. 
Cattin, M.-F., L.-F. Bersier, C. Banasek-Richter, R. Baltensperger, and J.-P. Gabriel. 2004. Phylogenetic constraints and adaptation explain food-web structure. Nature 427: $835-839$.

Cohen, J. E. 1977. Ratio of prey to predators in community food webs. Nature 270:165-167.

Cohen, J. E., F. Briand, and C. M. Newman. 1990. Community food webs: data and theory, Springer Verlag, Berlin, Germany.

Connor, E. F., and D. Simberlof. 1979. The assembly of species communities: Chance or competition? Ecology 60 1132-1140.

Fagan, W. F. 1997. Omnivory as a stabilizing feature of natural communities. American Naturalist 150:554-567.

Gale, D. 1957. A theorem on flows in networks. Pacific Journal of Mathematics 7:1073-1082.

Gotelli, N. J. 2001. Research frontiers in null model analysis. Global Ecology and Biogeography 10:337-343.

Holt, R. D. 1996. Community modules. Pages 333-349 in A. C. Gange and V. K. Brown, editors. Multitrophic interactions in terrestrial ecosystems. Blackwell Science, Oxford, UK.

Huxham, M., S. Beaney, and D. Raffaelli. 1996. Do parasites reduce the change of triangulation in a real food web? Oikos 76:284-300

Martinez, N. D. 1991. Artifacts or attributes? Effects of resolution on the Little Rock Lake food web. Ecological Monographs 61:367-392.

McCann, K., and A. Hastings. 1997. Re-evaluating the omnivory-stability relationship in food webs. Proceedings of the Royal Society of London B 264:1249-1254.

McCann, K., A. Hastings, and G. R. Huxel. 1998. Weak trophic interactions and the balance of nature. Nature 395 : 794-798

Melián, C. J. and J. Bascompte. 2004. Food web cohesion. Ecology 85:352-358.

Memmott, J., N. D. Martinez, and J. E. Cohen. 2000. Predators, parasitoids and pathogens: species richness, trophic generality and body sizes in a natural food web. Journal of Animal Ecology 69:1-15.

Milo, R., S. Itzkovitz, N. Kashtan, R. Levitt, S. Shen-Orr, I. Ayzenshat, M. Sheffer, and U. Alon. 2004. Superfamilies of evolved and designed networks. Science 303:15381542.

Milo, R., S. Shen-Orr, S. Itzkovitz, N. Kashtan, D. Chklovskii, and U. Alton. 2002. Network motifs: simple building blocks of complex networks. Science 298:824-827.

Pimm, S. L. 2002. Food webs. Second edition. The University of Chicago Press, Chicago, Illinois, USA.

Pimm, S. L. and J. H. Lawton. 1977. Number of trophic levels in ecological communities. Nature 268:329-331.

Pimm, S. L. and J. H. Lawton. 1980. Are food webs divided into compartments? Journal of Animal Ecology 49:879898.

Polis, G. A. 1991. Complex desert food webs: an empirical critique of food-web theory. American Naturalist 138:123155.

Polis, G. A., and R. D. Holt. 1992. Intraguild predation: the dynamics of complex trophic interactions. Trends in Ecology and Evolution 7:151-154.

Polis, G. A., and D. R. Strong. 1996. Food web complexity and community dynamics. American Naturalist 147:813846.

Reagan, D. P., and R. B. Waide. 1996. The food web of a tropical rain forest. University of Chicago Press, Chicago, Illinois, USA.

Roberts, A., and L. Stone. 1990. Island-sharing by archipelago species. Oecologia 83:560-567.

Sugihara, G., K. Schoenly, and A. Trombla. 1989. Scaleinvariance in food-web properties. Science 245:48-52.

Williams, R. J., and N. D. Martinez. 2000. Simple rules yield complex food webs. Nature 404:180-183.

Williams, R. J., and N. D. Martinez. 2004. Limits to trophic similarity and omnivory in complex food webs: theory and data. American Naturalist 163:458-468. 